\title{
AUTHOR INDEX VOLUME 9 (2007)
}

Arin, J., Egalitarian distributions in coalitional models

Basili, M. \& Fontini, F., Constitutional constraints under ambiguity: A game-theoretic approach

Benchekroun, H., A unifying differential game of advertising and promotions

Biswas, A. K., see Thangaraj, V.

Briec, W., Benefit function and duality in finite normal form games

Cartwright, E. J., Contagion and the speed of adjustment in small worlds

Côrte-Real, P. P., Fuzzy voters, crisp votes

Courtault, J.-M., Crettez, B. \& Hayek, N., A note on Luenberger's zero-maximum principle for core allocations

Crettez, B., see Courtault, J.-M.

De Marco, G. \& Morgan, J., A refinement concept for equilibria in multicriteria games via stable scalarizations

Do, K. H. P. \& Norde, H., The shapley value for partition function form games

Dulleck, U., The E-mail game revisited - Modeling rough inductive reasoning

Eriksson, K. \& Sjöstrand, J., On two theorems of Quinzii and rent controlled housing allocation in Sweden or "I buy your slithy tove only if my best friend's wife buys your best friend's husband's slithy tove"

Fontini, F., see Basili, M.

Fragnelli, V., Llorca, N. \& Tijs, S., Balancedness of the class of infinite permutation games and related classes of games

Gambarelli, G., Transforming games from characteristic into normal form

Gangopadhyay, P., Competitive tax evasion and transfer prices

Glizer, V. Y., see Turetsky, V.

Hayek, N., see Courtault, J.-M.

Hucki, Z. \& Kolokoltsov, V. N., Pricing of rainbow options: Game theoretic approach

Ju, Y., The consensus value for games in partition function form
1 (2007) $47-57$

1 (2007) 59-66

2 (2007) 183-197

2 (2007) 361-374

3 (2007) 495-513

4 (2007) 689-704

1 (2007) 67-86

3 (2007) 453-460

3 (2007) 453-460

2 (2007) 169-181

2 (2007) 353-360

2 (2007) 323-339

3 (2007) 515-525

1 (2007) 59-66

3 (2007) 425-435

1 (2007) 87-104

2 (2007) 347-351

4 (2007) 637-655

3 (2007) 453-460

2 (2007) 215-242

3 (2007) 437-452 
Kamphorst, J. \& van der Laan, G., Network formation under heterogeneous costs: The multiple group model

Karray, S. \& Zaccour, G., Effectiveness of coop advertising programs in competitive distribution channels

2 (2007) 151-167

Kikuta, K., Twisted dual games and their properties

2 (2007) 285-306

Kilani, M., Evolution and the complexity of finite automata

Koh, W. T. H., Welfare loss in linear price-preference procurement auctions

4 (2007) 719-730

Kojima, F. \& Takahashi, S., Anti-coordination games and dynamic stability

Kolokoltsov, V. N., see Hucki, Z.

Kooreman, P., see Schoonbeek, L.

Llorca, N., see Fragnelli, V.

Marchi, E., E-Points for diagonal games I

Marchi, E., The Centipede of Rosenthal

Mattos, C., Vertical foreclosure in telecommunications in the long run: Full interconnection quality foreclosure $\times$ sleeping patents

Meertens, M., Potters, J. A. M. \& Reijnierse, J. H., On bargaining sets in symmetric games

Méndez Naya, J. \& Méndez Naya, L., Tariff retaliation and the free trade argument

Méndez Naya, L., see Méndez Naya, J.

Monjardet, B., Some order dualities in logic, games and choices

Moreno-Ternero, J. D., Bankruptcy rules and coalitional manipulation

Morgan, J., see De Marco, G.

Nakanishi, N., Free entry, market size, and the optimistic stability

Norde, H., see Do, K. H. P.

Ohnishi, K., On the payoff representations of normal form games

Pensavalle, C. A. \& Pieri, G., Variational inequalities in Cournot oligopoly

Perakis, A. N., see Yang, Z.

Pieri, G., see Pensavalle, C. A.

Potters, J. A. M., see Meertens, M.

Reijnierse, J. H., see Meertens, M.

Roy, J., When aspiring and rational agents strive to coordinate

Schoonbeek, L. \& Kooreman, P., The impact of advertising in a duopoly game

4 (2007) 667-688

2 (2007) 215-242

4 (2007) 565-581

3 (2007) 425-435

4 (2007) 705-718

2 (2007) 341-345

3 (2007) 527-549

2 (2007) 199-213

4 (2007) 657-666

4 (2007) 657-666

1 (2007) 1-12

1 (2007) 105-118

2 (2007) 169-181

2 (2007) 243-268

2 (2007) 353-360

3 (2007) 477-482

4 (2007) 583-598

2 (2007) 269-284

4 (2007) 583-598

2 (2007) 199-213

2 (2007) 199-213

3 (2007) 461-475

$4(2007) 565-581$

Schwartz, J. A. \& Wen, Q., Wage negotiation under good faith bargaining

Sjöstrand, J., see Eriksson, K.

Sugumaran, A., see Thangaraj, V.

Szentes, B., Two-object two-bidder simultaneous auctions

Takahashi, S., see Kojima, F.

3 (2007) 551-564

3 (2007) 515-525

2 (2007) 361-374

3 (2007) 483-493

4 (2007) 667-688

Thangaraj, V., Sugumaran, A. \& Biswas, A. K., On bargaining based point solution to cooperative TU games

2 (2007) 361-374 
Tijs, S., see Fragnelli, V.

Turetsky, V. \& Glizer, V. Y., Robust solution of a time-variable interception problem: A cheap control approach

van Benthem, J., Rational dynamics and epistemic logic in games van der Laan, G., see Kamphorst, J.

Vannucci, S., Game formats as Chu spaces

Vasil'ev, V., Weber polyhedron and weighted shapley values

Wen, Q., see Schwartz, J. A.

Wiese, H., Measuring the power of parties within government coalitions

Yang, Z. \& Perakis, A. N., A modified duopoly game model in bulk commodity transportation markets

Zaccour, G., see Karray, S.
3 (2007) 425-435

4 (2007) 637-655

1 (2007) 13-45

4 (2007) 599-635

1 (2007) 119-138

1 (2007) 139-150

3 (2007) 551-564

2 (2007) 307-322

2 (2007) 269-284

2 (2007) 151-167 